\title{
Use of cerium nitrate nanoparticles in biological environment along with the factors effecting it; An empirical overview
}

\author{
Zarish Fatima ${ }^{1}$, Sameer Quazi $^{2}$, Shreelaxmi Gavas ${ }^{3}$, Tomasz M Karpinski ${ }^{4}$
}

1. Department of Biochemistry and Biotechnology, University of Gujrat, Hafiz Hayat Campus, Gujrat, Pakistan.

Email: xarishfatima@gmail.com

2. Founder \& CEO, GenLab BioSolutions Private Limited, Bangalore, Karnataka, India. 560043.

Email: Colonel.quazi@gmail.com

3. Clinical Analyst, Department of Life Sciences, GenLab Biosolutions Private Limited, Bangalore, Karnataka, India. (560043)

Email: Shreelaxmi012@gmail.com

4. Chair and Department of Medical Microbiology, Poznan University of Medical Sciences, Wieniawskiego 3, 61-712 Poznan, Poland.

Email: tkarpin@ump.edu.pl

\begin{abstract}
:
The advancement in the production and usage of the cerium oxide nanoparticles have diverted the attention of scientists towards their usage in medical field and therapeutic usage. The clinical usage of these Nano ceria is based on their ability to moderate the oxidative stress and this is only because of their ability to change their valent state from +3 to +4 which makes them ideal for scavenging radicals for use in a number of systemic and neurodegenerative disorders. This review aims to synthesize the basic methods used for the synthesis of nanoparticles along with the use of ligand, stabilizing agent and other components. This review also concludes that how various physical and chemical properties of nanoparticles effect the basic biological activities such as antimicrobial activity, cytotoxicity and many others. However, during the standardization, some of the physiochemical properties, methods used for preparation and catalytic abilities must be taken into account.
\end{abstract}

Keywords:

Cerium oxide nanoparticles, radicals, oxidative stress, physical and chemical properties and biological characteristics. 


\section{1) Introduction:}

A nanoparticle is a very small entity having the size range from 1 to $100 \mathrm{~nm}$, usually found to be undetectable by the naked eye and exhibit much difference in the term of properties both physical and chemical in comparison to their smaller counter parts. They possess the atomic size of about 0.1 meter and their size continue to change as they reach to the size of atom [1]. The fact behind is the increased surface to volume ratio that ultimately results in the dominance of material surface properties as compared to its performance [2]. Based on their size, these nanoparticles are thought to possess very high charge to surface ratio as compared to much larger objects such as sheets or powders etc. These properties thus enable these nanoparticles to possess certain physical, chemical and optical properties as they are small enough to confine their effects in the forms of electrons to produce the quantum effects. Metallic nanoparticles have fascinated the science over the last few decades and now is overcoming the both biomedical and engineering fields [3]. They are of great value because of their huge part in the field of nanotechnology. These days these nano particles have been modified with number of the chemical functional groups in order to acquire certain properties like physiochemical properties and biological attributes to be used in the field of diagnosis and treatment. There are a number of metal-based nanoparticles which are used for the biomedical applications which include iron, copper, silver, gold and many others. But the cerium nanoparticles have dominated all of them in their applications. Cerium belongs to the lanthanide series in the periodic table which exists in fluorite structure in the form of oxides. Even in the case of the deficiency of oxygen, they retain their form of fluorite structure[4]. This results in the production of cerium oxide nanoparticles being used for a number of oxidoreduction reactions. This catalytic property is determined by the specific arrangement of fluorite in cerium oxide nanoparticles. These all characteristics allow the cerium to be used in the industry as for example in the reduction or removing of the carbon monoxides or other carbon-based hydrocarbons from the exhaust gases. This is further has ignited by the existence of the metal i.e. cerium [5]. These nanoparticles formed by cerium mitigate certain properties such as it helps in the tolerance of the oxidative stress found to be associated with a number of nervous system disorders such as Parkinson disease or Alzheimer disease [6]. These nanoparticles also function in identical to a number of enzymes such as superoxide dismutase, catalases, phosphatases and oxide reductase on the basis of their switch in the valences [7]. 
Moreover, these nanoparticles are very cheap and have the property to tolerate very harsh conditions because of their characteristics. They are found to poses very strong SOD like properties although they are not very powerful enzymes occurring in naturally prevailed conditions. They function as super oxide dismutase by the scavenging of the most reactive oxygen species that also make them biocompatible for use [8]. Their enzymatic properties are also dependent on surface area properties. Another research has demonstrated that these nanoparticles oxidation using hydrogen peroxide has resulted in decreased activity as compared to others when viewed with photoelectron spectroscopy. It also decreases the ratio of $\mathrm{Ce}+3$ to $\mathrm{Ce}+4$ which is directly related to the loss of mimetic activity of SOD. On the other hand, it also possesses catalase mimicking activity which has shown the mimicking activity of these nanoparticles with that of when treated with pulsed electron evaporation. These properties were measured by the addition if the hydrogen peroxide to cerium oxide nanoparticles and the absorbance was measured at 380nm. Vazirov and his colleagues have doped the cerium oxide nanoparticles with other metals which increases or alters the ratio of $\mathrm{Ce}+3$ to $\mathrm{Ce}+4$ and also the valences on surface of nanoparticles. This in turn also increases the rate of reaction and also the point of interaction. These catalase dependent properties depend upon concentration of $\mathrm{Ce}+3$ [9]. Amplex red assay was used for the accessing of cerium oxide concentration and also cerium like activities. The test demonstrated the high level of catalase activity in the solution containing $\mathrm{Ce}+4$ as compared to $\mathrm{Ce}+3$. In general, the two mechanisms which play central role in the regulation of cerium nanoparticles include phosphorylation and dephosphorylation. This involves the addition or removal of phosphate resulting in production in storage of energy. So it is of prime importance in the synthesis of ATP while phosphate is used for a number of cellular and metabolic reactions [10]. Cerium oxide nanoparticles were tested for phosphate type activity in another study. It also decreases the level of ATPase and it also exhibit decrease in the viability of cell by increase production of ADP which causes increased level of toxicity[11]. 


\section{2) General procedure for the synthesis of nanoparticles:}

There exist a number of techniques which are used for the preparation of cerium oxide nanoparticles from various methods. These include hydrothermal, solvent extraction, reversed precipitation, reversed micelle formation, flame spray for the preparation of nanoparticles on the basis of their size and other properties. The synthesized compounds are found to be remain in their natural form or they can be coated with other substances which can either be hydrophilic or hydrophobic. For the chemical synthesis of nanoparticles the salt of cerium nitrate is mixed in the deionized water and an equal amount of oxalic acid is poured in it in order to act as a fuel [12]. Upon heating, calcination and sintering it result in the formation of cerium oxide nanoparticles in spherical shape. On the other hand, or the biological synthesis the plant extract or pure water is used which later results in the synthesis of nanoparticles. The protection used in this case can either be glycol, dextran, polyglucans and glucose [13]. The size is of much importance because they are helpful in the maintenance of the properties of nanoparticles such as surface area, size, charge, structural morphology and other characteristics of the nanoparticles. One of the most important property is the catalytic activity. These properties can be controlled and analyzed in a specific manner in order to meet the requirements for the specific applications [14].

\section{3) Basic size of nanoparticles and its effect on biological properties}

Most probably, the size of nanoparticles allows the riper delivery of drug to the site of infection. Thus, enabling the crossing of transmembrane barrier. So, the size of the nanoparticles must not be altered in order to get certain properties. Any change in the size can further influence other properties of the nanoparticles such as immunological properties, half-life and diversity. A certain reduction in surface to volume ratio causes increase in the size of nanoparticle [15]. With the decrease in the size of nanoparticle, an increase in the oxygen carrying capacity takes place. Increase in the level of oxygen causes the increment in the increase in the movement of oxygen through crystal lattice resulting in the more ability of nanoparticle to oxidize or reduce the substances. This in turn gives the results that decrease in the cerium nanoparticle has resulted in the reduced or more expansion of lattice showing direct relationship between two variables. This in turn has resulted in the rearrangement of the valences of oxygen on the surface of nanoparticles [16]. 
Morens and his colleagues have cleared this fact by putting forward the idea that these cerium oxide nanoparticles have shown extra potential for the anti-bacterial activities as compared to the silver nanoparticles. Similarly, these cerium oxide nanoparticles are shown to exhibit more powerful anti-oxidative potential as compared to other when their doping is performed with the metal gadolinium during magnetic resonance imaging being abbreviated as MR. Zhang and his colleagues was successful in preparation of the cerium oxide nanoparticles by the adjustment of the certain parameters. As for example in the scenario of the preparation of nanoparticles with the small size they tried to adjust the reaction time or rate. While for the preparation of the nanoparticles of charger size, they increased the temperature from $500-850^{\circ} \mathrm{C}$ for about 40 minutes. Self and his colleagues have suggested that the interaction of biological entities with the cerium oxide nanoparticles is required for the therapeutic purpose usage. Similarly, the size of the nanoparticles helps in the clearance of these therapeutic particles from the immune system, thus resulting in the vascular clearance of these particles. Smaller nanoparticles gets clear more rapidly or early as compared to larger ones which tend to accumulate [17]. However, of we add the satirizer molecules to the nanoparticles, then this accumulation can be prevented. Also, it also results in the increased retention time of the nanoparticle containing drug. Thus the clearance of the accumulated nanoparticles caused by the aggregation is as a result of reticulum endothelium clearance. On the other hand Xu has suggested that human body don't possess any type of mechanism required for the removal of these cerium oxide nanoparticles from the body as these type of the metallic nanoparticles are not produced naturally in the body [18]. On the other hand, there are the chances that these nanoparticles can be ingested when taken in the form of tablet and can be cleared by the excretory system in a proper way. As for example the nanoparticles of the size as smaller than that of $5 \mathrm{~nm}$ can be excreted by the excretory or renal system which is also a negatively charged particle. In the case of the size of cerium oxide the particles having smallest size i.e. about $3 \mathrm{~nm}$ are more active as compared to larger ones in the term of toxicity [19]. The lower toxicity in the term of smallest nanoparticles is due to the fact that they show phenomenon of algomerization. Om the other hand, the larger nanoparticles of cerium oxide has shown much more toxicity as compared to smaller ones. But in the scenario of Kinetic analysis these nanoparticles have shown to be less toxic. The preservation of these nanoparticles in soil has utmost chances to catch microbes $[\underline{19}$, 20]. 


\section{4) Physical structure or properties of nanoparticles:}

The morphology or the structure of the nanoparticles is one of the most important properties when considering the pharmacological related properties. The morphology of the nanoparticle is the most important thing when we study the relationship between the nanoparticles and biological system. Nanoparticles can exist in a number of shapes or structures such as spherical, cuboidal, rectangular, rod shaped and in the polygonal form [21]. Nanoparticles having the sharpest edges have the chances to disrupt the membranes of biological system due to its sharper edges. Hence these are the nanoparticles are found to be less compatible in the biological environment. So the size and structural shape of nano particles play a much beneficial and crucial role in the fate and the behavior of these freshly prepared nanoparticles in the biological system. This can also be due to difference in the charge and diffusion rate of nanoparticles [22]. Control on the morphological properties help them to fit in the various properties and emerging technologies. As for example they are used in the optical fibers and plasma technology in order to attain the property of anisotropy to give losses of plasmon. In spite of the basic morphology of nanoparticles, it is not found to be fully characterized and is uncontrollable [23]. The structural characteristics which need to be taken into account include flat shaped, spherically arranged and ratio of aspects. Hence it play an important role in the fate and behavior of the nanoparticles in the environment. This in turn is totally based on the difference in the rate of diffusion which changes with the aspect ratio of the material. A number of the reports have published which have given the certain evidences on the role of shape and size on the internalization of cell. As it is much easier to produce via hydrothermal process but the synthetic conditions must be carefully controlled in order to obtain the nanoparticles of specific morphology [24]. However, there are certain challenges which are faced by this solvent or hydrothermal process. The black box reaction needs to be understand before the preparation. Hence reaction conditions must be access before. Secondly it is very difficult to synthesize these nanoparticles on the larger scale because of the much harsh conditions which need to be maintained during the synthesis [25].

Another fact if which need to be considered in the terms of cerium nitrate nanoparticles is their shape which play a key role in the toxicity. It has been found that the translocation ratio of the nanoparticles can be increased up to $95 \%$ based on their shape and surface charge [26]. It has also been found that these cerium nanoparticles can increase the toxicity in the macrophages obtained 
from a specific cell line. While the nanoparticles which are cubic or octahedral were not found to display any other such property. Similarly in another research in 2018 , it has been elucidated that theses octahedral and cubic nanoparticles have potential against the carcinoma cells in human body. The cube and shaped nanoparticles have exhibited relative lower toxicity. These all properties of Nano ceria are based on the type of cell and biological response shown by them [27].
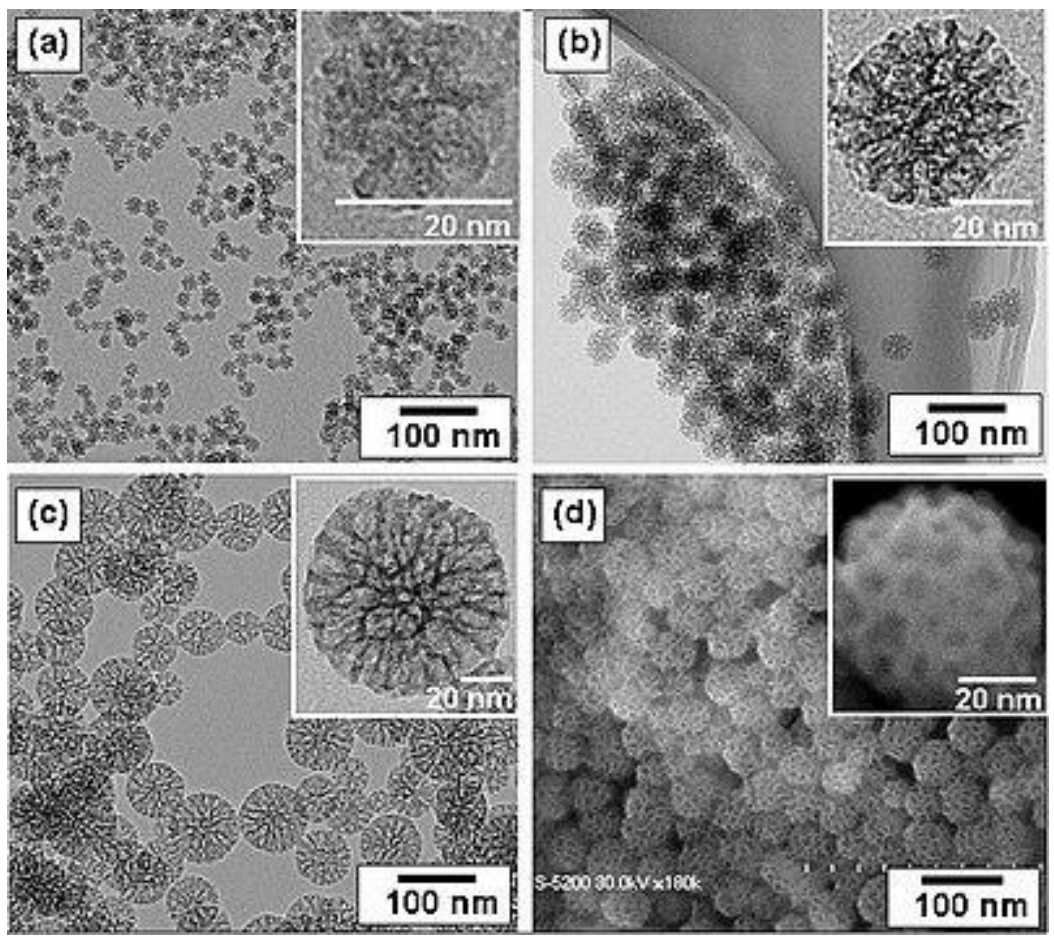

Fig.01. Morphological structure of nanoparticles along with size range

\section{5) Physical properties of nanoparticles:}

There are certain nanoparticle physical characteristics which are accessed during the synthesis such as redox potential which is helpful in providing specific shape to the crystal structure of nanoparticles. As we are aware of the fact that cerium exists in both forms either trivalent or tetravalent which needs to switch between each other in order to attain a specific shape of crystals. This low energy alternations give specific properties to the crystal structure of cerium oxide nanoparticles. However it is taken into account that these oxidation state varies among each of the individual nanoparticle resulting in the variation of valence in both for dither +3 or +4 state and is totally dependent on the size of the nanoparticles [28]. So it is much imperative to study or investigate the redox reaction condition of the $\mathrm{CeO}$ nanoparticle. Similarly, diffusion is another 
process which involve the movement of the dissolving substances from area of lower concentration to area of higher concentration via diffusion process. The temperature-based parameter which tends to control this process is termed as solubility. Metal based nanoparticles which in this case can either be zinc oxide or cerium oxide have the ability to dissolve quickly which in turn results in the toxicity effects [29]. The dissolution limit and the to in the term of the nano particle and its dissolved form both need to be considered for the better understanding of the use of nanoparticles in use for organisms. Similarly, the fractionation of the surface of the nanoparticles has been employed for the synthesis of the less toxic, biocompatible and biodegradable type nanoparticles. So that both form of therapeutics either water soluble or insoluble form of therapeutics can be used in the patients having any sort of ailment. As in the care of the cerium oxide nanoparticle preparation, s number of the polymers have been used in order to obtain these particles in different forms based on the surface to charge ratio [30]. Moreover, these cerium, oxide nanoparticles are covered with a layer of hydroxyl group so these biodegradable, biocompatible and hydroxyl possessing nanoparticle small moieties have tendencies to stabilize the nanoparticle structures [31].

The application of these nanoparticles with the polymers or other stabilizing agent can aid in the controlling of the diameters of these nanoparticles. But the major challenge faced by the nano particles here is their ability to maintain the physiochemical properties. The surface charge or the lower surface to volume ratio of these nanoparticles often results in the algomerization of these article and thus increases the range if these particles from the nano range to the micro range which often results in the major problems in the term of their usage as an effective therapeutic agent [32]. So in order to combat this state the surface coating is often employed in order to obtain the conditions where there are no chances of the oligomerization of the nanoparticles. The coating also increases the chances of the solubility of these nanoparticles. In addition to it, also it can be employed for the minoring the properties of toxicities which leaves a promising effect in therapeutics [33].

\section{6) Chemical properties and their effect on biological properties:}

The chemical characteristics of the nanoparticles is found to play a crucial role in the parchment of the surface charge on the basis of which the nanoparticles agglomerate together which in turn effect the solubility of the nanoparticles. In order to prevent the instability of the suspension, most 
of the nanoparticles are coated with the certain type of surfactants [34]. The presence of the surface charge on the nanoparticles can modify their surface properties to a significant extent as compared to uncoated nanoparticles. As for example in the case of Pristine the similar outcomes were observed when they are coated with humic acid. So the coating of the humic acid on the surface of the silver nanoparticles result in the disassembly or aggregation of the nanoparticles [35]. On the other hand, the authors also have the suggestion that oligomerization has further been further reduced by the application of the stabilizers on the surface of the nanoparticles which has also reduced the toxicity based on algomertization.In the case of cerium oxide nanoparticles, the environment which surrounds the cerium nanoparticles also effects their surface chemistry. So the reaction of the oxygen with the hydrogen and with the solution has resulted also in the alternation of the chemistry of surface of nanoparticles which also effects their catalytic ability in return also effects their catalytic ability [36]. A very strong oxidizing agent formed by the reaction of the superoxide and nitric oxide based redials is peroxynitrate. In addition to their ability of the reduction of nitro tyrosine to about $75 \%$, the Nano cerium has the ability to react with peroxynitrate. The specie is found to be stable under both acidic and alkaline conditions. But it can also decomposes abruptly or instantaneously at natural PH [37]. This has allowed there action if the chemical with Nano ceria. In the case when the Nano ceria is present at the much natural $\mathrm{PH}$, the decomposition of the peroxynitrate has been speed up. At the same time uric acid, nitric acid has accelerated the decay at the same time. So it has been found that this Nano ceria has the capability to react with peroxynitrate in vivo conditions. It has also shown to reduce the level of nitro tyrosine when they are exposed to peroxynitrate which also shows the results in turn. But care must be taken as when the Nano ceria reacts with the peroxynitrate, it result in the production of the certain redials[38].

\section{7) Effect of surface charge on the biological properties of Nano ceria:}

The surface charge on the nanoparticle surface determines the various biological properties of nano particles such as respiration rate, cellular uptake, distribution in the biological environment and its interaction with the biology environment at any level and can be found by the relative position to zeta potential [39]. If in any case the deposition occurs at the negative side, the surface charge on nanoparticle will also be negative. While on the other hand if it lies on the positive side then the surface charge will also be positive. So the surface charge is found to have a very strong impact 
on the interaction of the nanoparticles with the biological environment. The most commonly controlled mechanisms in the term of the control by surface charge include the ability of nanoparticles to cross the blood brain barrier, binding of the plasma protein and the transmembrane permeability [40]. The dissolution and aggregation of the cerium oxide nanoparticles is dependent on its surface charge. They were found to be functionalized or exhibit three types of charges based on the coatings such as positive, negative and neutral charges. And on the basis of this charge, they exhibit certain responses to toxicity. The more positive nanoparticles are found to exhibit more toxicity activity as compared to the negative nanoparticles. Cytotoxicity of the cerium, oxide nanoparticles in the term of the microbes is elicited by a group of researches where it has exhibited antimicrobial activity and the smaller nanoparticles showed more antimicrobial properties as compared to larger ones [35]. However, in the presence of normal saline solution, these positively charged nanoparticles have exhibited its direct attack on the cell membrane and cell wall in the term of plant which tends to destabilize it and in turn increases the production of reactive oxygen species being abbreviated as ROS. On the other hand in the same case, the cytotoxicity cannot be determined [41].

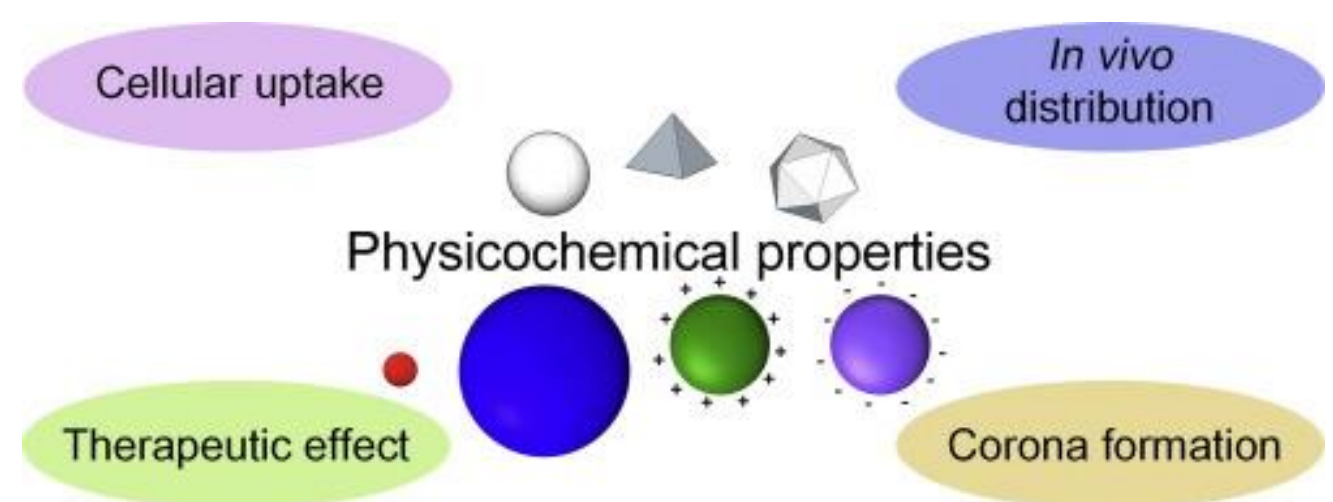

Fig. 02.The effect of surface charge on the biological properties exhibited here.

\section{8) Effect of agglomeration and surface coating on biological characteristics of Nano ceria:}

Agglomeration is the most basic process which results in the reduction in the surface charge and increase in the size of nanoparticles. This can be resulted from the combination of the nanoparticles via weak forces leading to the creation of sub micronized entities. It has the ability to change physical properties of nanoparticles which in turn effect their biological activity [42]. The aggregation of the number of nanoparticles depends upon their certain properties such as size, 
shape, $\mathrm{PH}$, organic matter present in natural form. On the other hand increasing the NOM concentration can increase the removal of chances of agglomeration to about $85 \%$. The much lower toxicity id observed in the term of much higher adsorption properties of NOM at very lower PH for cerium nitrate [43]. The type of adsorption not only inhibits the interaction or interrelation of the nanopsrticles with the others but it also results in the reduction of the cerium oxide nanoparticle properties with itself and other cells which also reduce its bioavailability. It is also found that the agglomerated form of the nanoparticles is shown to have much more toxicity as compared to others. While the dispersed nanoparticles haven't shown any such effects at all. Some of the cerium based nanoparticles along with its host and their effect on biological function is depicted in table $01[44]$.

Similarly, the surface coating of nanoparticles also play a major role in the toxicity. The surface coating cover the surface area of nanoparticles and tend to provide them the stabilization in order to prevent agglomeration. It also imparts certain properties which include increased solubility of the nanoparticles. Mostly they are covered by the poly acrylic acid and their stability is found to comparable with pristine form [45]. They have also found to increase the solubility or stability based on the increased form of pristine. As a study in 2017 has indicated that coating of the nanoparticles with silica has resulted in reduced inflammatory responses of pulmonary system. When this Nano ceria are reacted with citrate, it results in the formation of the nanoparticles with the property that they interact with cell in order to make its cells or compartments stronger. So in the presence of higher concentration, these citrate containing cerium nanoparticles have shown a higher level of toxicity but in addition also shows moderate level of genetic toxicity lastly those coated with poly acrylic acid PAA did not depict any sort of toxicity [46]. 


\begin{tabular}{|c|l|l|}
\hline \multicolumn{1}{|c|}{ Nanoparticles } & \multicolumn{1}{|c|}{ Size } & \multicolumn{1}{c|}{ Biological activity } \\
\hline $\mathrm{CeO}_{2}$ & \multicolumn{1}{|c|}{ Less than 25nm } & Activation of MAPK pathway \\
\hline $\mathrm{CeO}_{2}$ & About $4 \mathrm{~nm}$ & Cytotoxicity in cancer cell \\
\hline $\mathrm{CeO}_{2}$ quantum dots & $7 \mathrm{~nm}$ & Anti-oxidant \\
\hline $\mathrm{CeO}_{2}$ nanotubes & $10-20 \mathrm{~nm}$ & Antimicrobial effects \\
\hline $\mathrm{CeO}_{2}$ Nano dots & $20 \mathrm{~nm}$ & Antibacterial activity \\
\hline $\mathrm{Neutron}^{2}$ activated $\mathrm{CeO} 2$ & $41 \mathrm{~nm}$ & $\begin{array}{l}\text { Left bronchial instillation } \\
\text { Activation of } \\
\text { pathways }\end{array}$ \\
\hline $\mathrm{CeO}_{2}$ hexa hydrate & $10 \mathrm{~nm}$ & \\
\hline
\end{tabular}

\section{9) Effect of dissolution and oxygen vacancy on Nano cerium properties:}

For toxicity purposes, it is crucial to study the effect of desolation or valence bonds of oxygen in order to have idea about vacancy. It is found that the cerium having valence 3 is much more stable and effective as compared to other cerium oxide nanoparticles [47]. Many studies has indicated that the dissolution of cerium in the form being available in biological system is much dependent on PH. The phenomenon of dissolution was observed at the acidic PH of less 7 resulting in the increased level of acidity. It is also found that the phosphate ion could reduce the process of dissolution cue to its high affinity and desolation on the surface of nanoparticles [48]. This is the most important process to be observed in the term of toxicity of cerium oxide nanoparticles. Phosphate ligands can inhibit the desolation as compared to its other counterparts. This is considered to be the most important thing in keeping the regulation of the environment using cerium nitrate nanoparticles. The use of the agglomerated nanoparticles is much toxic for the sake of green algae as compared to when they are present in their dispersed form. The difference can attributed to the different separation of the nanoparticle sample in the suspension. In dispersed form, cerium oxide nanoparticles agglomerate and get dissolved in the trivalent form so that they are not available for the bioavailability [49].

The valence of cerium can also change the toxicity properties of cerium nanoparticles. When these nanoparticles are doped with $\mathrm{Gd}_{2} \mathrm{O}_{3}$, it results in the fluctuance in between two valent states $\mathrm{Ce}+3$ and $\mathrm{Ce}+4$. The doping has resulted in the increased chances of cerium to agglomerate and also enhances the valence state and oxygen vacancy of cerium oxide nanoparticles [50]. However, in 
the case of the pulmonary toxicity and other disorders. The process is surprising because the tendency of the cerium to jump between two valences is very low. A large number of the applications of cerium nitrate has indicated their use in a number of the texological evolutions [51]. A number of the extensive studies have been performed in order access to the toxic effects. Higher vacancies of the cerium nitrate has resulted in the change in the reduction oxidation potential of nanoparticles synthesized by them [52]. These all things in turn effect the toxicity as for example physiochemical properties of nanoparticles, cell lines, their route of exposure, targeted organism and the type of studies either in vivo or in vitro. So a lot of confusing and sometimes much contradicting data is available in order to obtain a comprehensive view [53]. So in this attempt we are classifying the ethological studies based on their toxic assessment such as cytotoxicity, respiratory toxicity, toxicity based on liver, phytotoxicity and environmental toxicity. This type of classification helps scientists to understand and overcome the serious effects of cerium oxide nanoparticles $[54,55,56,57]$.

\section{0) Conclusion:}

These studies have revealed the therapeutic effects of cerium nitrate nanoparticles in the biological environment. This has also elucidated that how surface properties, such as charge, size, volume has affected the synthesis and applications pf these cerium oxide particles in biological system. Moreover, the importance of the better synthesis method along with the modifications using certain ligands is also demonstrated. The effect of certain stabilizers, ligands and adjuvants is clearly found that how it effects the properties of synthesized nanoparticle structure. In the term of their usage in the human system, these nanoparticles are synthesized in a biologic manner to avoid immunological or allergic reactions in human body. Because most of the past studies have shown that these nanoparticles have caused inflammation, oxidative stress and bacteriological infection. So, there is a need to further explore the science of these nanoparticles so that they can further be used in the diseases which require tissue regeneration. 


\section{References:}

1. Mody, V.V., et al., Introduction to metallic nanoparticles. Journal of Pharmacy and Bioallied Sciences, 2010. 2(4): p. 282.

2. Moores, A. and F. Goettmann, The plasmon band in noble metal nanoparticles: an introduction to theory and applications. New Journal of Chemistry, 2006. 30(8): p. 1121-1132.

3. Holister, P., et al., Nanoparticles. Technology white papers, 2003. 3: p. 1-11.

4. Rotello, V., Nanoparticles: building blocks for nanotechnology. 2004: Springer Science \& Business Media.

5. Zabirnyk, O., M. Yezhelyev, and O. Seleverstov, Nanoparticles as a novel class of autophagy activators. Autophagy, 2007. 3(3): p. 278-281.

6. Li, Y.-Q., et al., Facile synthesis of highly transparent polymer nanocomposites by introduction of core-shell structured nanoparticles. Chemistry of Materials, 2008. 20(8): p. 2637-2643.

7. Rai, M. and N. Duran, Metal nanoparticles in microbiology. 2011: Springer Science \& Business Media.

8. Miura, M., et al., Enhancement of flux-pinning in epitaxial Sm1 $+x B a 2-x C U 3 O y$ films by introduction of low-Tc nanoparticles. Japanese journal of applied physics, 2005. 45(1L): p. L11.

9. Fedlheim, D.L. and C.A. Foss, Metal nanoparticles: synthesis, characterization, and applications. 2001: CRC press.

10. Vollath, D., Nanomaterials an introduction to synthesis, properties and application. Environmental Engineering and Management Journal, 2008. 7(6): p. 865-870.

11. Gubin, S.P., Magnetic nanoparticles. 2009: John Wiley \& Sons.

12. Hong, C.-S., et al., Effect of metal (Al, Ga, and In)-dopants and/or Ag-nanoparticles on the optical and electrical properties of ZnO thin films. Thin Solid Films, 2006. 515(3): p. 957-960. 13. Louis, C. and O. Pluchery, Gold nanoparticles for physics, chemistry and biology. 2017: World Scientific.

14. Bandeira, M., et al., Green synthesis of zinc oxide nanoparticles: A review of the synthesis methodology and mechanism of formation. Sustainable Chemistry and Pharmacy, 2020. 15: p. 100223.

15. Kharissova, O.V., et al., Greener synthesis of chemical compounds and materials. Royal Society open science, 2019. 6(11): p. 191378.

16. Castillo-Henríquez, L., et al., Green synthesis of gold and silver nanoparticles from plant extracts and their possible applications as antimicrobial agents in the agricultural area. Nanomaterials, 2020. 10(9): p. 1763.

17. Estevez, M.B., et al., Biofilm eradication using biogenic silver nanoparticles. Molecules, 2020. 25(9): p. 2023.

18. Hamida, R.S., et al., Synthesis of silver nanoparticles using a novel cyanobacteria Desertifilum sp. extract: their antibacterial and cytotoxicity effects. International journal of nanomedicine, 2020. 15: p. 49.

19. Youssif, K.A., et al., Anti-Alzheimer potential, metabolomic profiling and molecular docking of green synthesized silver nanoparticles of Lampranthus coccineus and Malephora lutea aqueous extracts. PloS one, 2019. 14(11): p. e0223781.

20. Zhang, F., et al., Cerium oxide nanoparticles: size-selective formation and structure analysis. Applied physics letters, 2002. 80(1): p. 127-129. 
21. Celardo, I., et al., Pharmacological potential of cerium oxide nanoparticles. Nanoscale, 2011. 3(4): p. 1411-1420.

22. Das, S., et al., Cerium oxide nanoparticles: applications and prospects in nanomedicine. Nanomedicine, 2013. 8(9): p. 1483-1508.

23. Asati, A., et al., Oxidase-like activity of polymer-coated cerium oxide nanoparticles. Angewandte Chemie, 2009. 121(13): p. 2344-2348.

24. Dowding, J.M., et al., Cerium oxide nanoparticles scavenge nitric oxide radical ( $\left.{ }^{*} \mathrm{NO}\right)$. Chemical communications, 2012. 48(40): p. 4896-4898.

25. Wason, M.S. and J. Zhao, Cerium oxide nanoparticles: potential applications for cancer and other diseases. American journal of translational research, 2013. 5(2): p. 126.

26. Lin, W., et al., Toxicity of cerium oxide nanoparticles in human lung cancer cells. International journal of toxicology, 2006. 25(6): p. 451-457.

27. Rajeshkumar, S. and P. Naik, Synthesis and biomedical applications of cerium oxide nanoparticles-a review. Biotechnology Reports, 2018. 17: p. 1-5.

28. Park, E.-J., et al., Oxidative stress induced by cerium oxide nanoparticles in cultured BEAS-2B cells. Toxicology, 2008. 245(1-2): p. 90-100.

29. Gao, Y., et al., Cerium oxide nanoparticles in cancer. OncoTargets and therapy, 2014. 7: p. 835 .

30. García, A., et al., Acute toxicity of cerium oxide, titanium oxide and iron oxide nanoparticles using standardized tests. Desalination, 2011. 269(1-3): p. 136-141.

31. Pelletier, D.A., et al., Effects of engineered cerium oxide nanoparticles on bacterial growth and viability. Applied and environmental microbiology, 2010. 76(24): p. 7981-7989.

32. Asati, A., et al., Surface-charge-dependent cell localization and cytotoxicity of cerium oxide nanoparticles. ACS nano, 2010. 4(9): p. 5321-5331.

33. Hirst, S.M., et al., Bio-distribution and in vivo antioxidant effects of cerium oxide nanoparticles in mice. Environmental toxicology, 2013. 28(2): p. 107-118.

34. Patil, S., et al., Protein adsorption and cellular uptake of cerium oxide nanoparticles as a function of zeta potential. Biomaterials, 2007. 28(31): p. 4600-4607.

35. Wu, J., et al., Toxicity of two different size ceria nanoparticles to mice after repeated intranasal instillation. Journal of nanoscience and nanotechnology, 2019. 19(5): p. 2474-2482. 36. Colon, J., et al., Protection from radiation-induced pneumonitis using cerium oxide nanoparticles. Nanomedicine: Nanotechnology, Biology and Medicine, 2009. 5(2): p. 225231.

37. Hirst, S.M., et al., Bio-distribution and in vivo antioxidant effects of cerium oxide nanoparticles in mice. Environmental Toxicology, 2013. 28(2): p. 107-118.

38. Bao, Q., et al., Simultaneous blood-brain barrier crossing and protection for stroke treatment based on edaravone-loaded ceria nanoparticles. ACS nano, 2018. 12(7): p. 67946805.

39. Niu, X., J. Chen, and J. Gao, Nanocarriers as a powerful vehicle to overcome blood-brain barrier in treating neurodegenerative diseases: Focus on recent advances. Asian journal of pharmaceutical sciences, 2019. 14(5): p. 480-496.

40. Ghalamfarsa, G., et al., Application of nanomedicine for crossing the blood-brain barrier: Theranostic opportunities in multiple sclerosis. journal of immunotoxicology, 2016. 13(5): p. 603-619. 
41. Lu, X., et al., Development of L-carnosine functionalized iron oxide nanoparticles loaded with dexamethasone for simultaneous therapeutic potential of blood brain barrier crossing and ischemic stroke treatment. Drug Delivery, 2021. 28(1): p. 380-389.

42. Mendiratta, S., et al., Multidisciplinary role of mesoporous silica nanoparticles in brain regeneration and cancers: from crossing the blood-brain barrier to treatment. Particle \& Particle Systems Characterization, 2019. 36(9): p. 1900195.

43. Sintov, A., et al., Metal nanoparticles as targeted carriers circumventing the blood-brain barrier. International review of neurobiology, 2016. 130: p. 199-227.

44. Hardas, S.S., et al., Rat brain pro-oxidant effects of peripherally administered $5 \mathrm{~nm}$ ceria 30 days after exposure. Neurotoxicology, 2012. 33(5): p. 1147-1155.

45. Cox, A., et al., Protein-functionalized nanoparticles derived from end-functional polymers and polymer prodrugs for crossing the blood-brain barrier. European Journal of Pharmaceutics and Biopharmaceutics, 2019. 142: p. 70-82.

46. Wang, Y., J. Luo, and S.-Y. Li, Nano-curcumin simultaneously protects the blood-brain barrier and reduces $M 1$ microglial activation during cerebral ischemia-reperfusion injury. ACS applied materials \& interfaces, 2019. 11(4): p. 3763-3770.

47. Rzigalinski, B.A., C.S. Carfagna, and M. Ehrich, Cerium oxide nanoparticles in neuroprotection and considerations for efficacy and safety. Wiley Interdisciplinary Reviews: Nanomedicine and Nanobiotechnology, 2017. 9(4): p. e1444.

48. Martinelli, C., et al., Antioxidants and nanotechnology: promises and limits of potentially disruptive approaches in the treatment of central nervous system diseases. Advanced healthcare materials, 2020.9(3): p. 1901589.

49. Arya, A., et al., Cerium oxide nanoparticles promote neurogenesis and abrogate hypoxiainduced memory impairment through AMPK-PKC-CBP signaling cascade. International journal of nanomedicine, 2016. 11: p. 1159.

50. Yu, H., et al., ROS-responsive nano-drug delivery system combining mitochondriatargeting ceria nanoparticles with atorvastatin for acute kidney injury. Theranostics, 2020. 10(5): p. 2342.

51. Re, F., M. Gregori, and M. Masserini, Nanotechnology for neurodegenerative disorders. Maturitas, 2012. 73(1): p. 45-51.

52. Tamil Selvan, S., P. Padmanabhan, and B.z. Zoltán Gulyás, Nanotechnology-Based Diagnostics and Therapy for Pathogen-Related Infections in the CNS. ACS chemical neuroscience, 2019. 11(16): p. 2371-2377.

53. Gallardo-Toledo, E., C. Velasco-Aguirre, and M.J. Kogan, Inorganic Nanoparticles and Their Strategies to Enhance Brain Drug Delivery, in Nanomedicines for Brain Drug Delivery. 2021, Springer. p. 149-172.

54. Liu, Y., et al., Near-Infrared Radiation-Assisted Drug Delivery Nanoplatform to Realize Blood-Brain Barrier Crossing and Protection for Parkinsonian Therapy. ACS Applied Materials \& Interfaces, 2021.

55. Fatima, Z., \& Quazi, S. (2021). Use of nanomaterials for the immobilization of industrially important enzymes. European Journal of Biological Research, 11(3), 381-391.

56. Quazi, S. (2021). Vaccine in response to COVID-19: Recent developments, challenges, and a way out. Biomedical And Biotechnology Research Journal (BBRJ), 5(2), 105-109. Doi: 10.4103/bbrj.bbrj_166_20 
57. Gavas, S., Quazi, S., \& Karpiński, T. (2021). Nanoparticles for Cancer Therapy: Current Progress and Challenges. Preprints.Org. https://doi.org/10.20944/preprints202108.0218.v1 